\title{
DESSICATION KERATITIS
}

\author{
BY \\ Surgeon Lieut.-Commander J. E. M. AyoúB \\ R.N.V.R.
}

THIS paper describes a corneal affection which was first noticed amongst the troops of the Eighth Army fighting in the desert during the summer of 1942. The disease is new to ophthalmologists serving in the Middle East Forces, and service conditions have hindered the full investigation and follow-up of cases. It is hoped that this report will lead to general recognition of the existence of the disease so that it may be more fully investigated and prevented.

Incidence.-Most of the cases commenced, according to the histories, during certain periods, after heavy and prolonged fighting had broken out in Libya and the Western Desert in May, 1942.

Judged from the records of this Military Hospital the outstanding dates of onset were (1) the first ten days of June; (2) midJune; (3) the first week of July. After this there was a gap until October, when two cases occurred.

During the first two summers of the war in the desert no cases were seen. Had any cases been evacuated from the desert in 1940 they would probably have passed through this hospital. In 1941, with greater organisation of advanced ophthalmic centres, they might not have reached here. As methods of examination have not changed, it is unlikely that cases have passed unrecognised in this clinic before 1942. On the other hand, during and after the retreat on the Alamein positions this hospital was close to the line and on an easy line of evacuation for sick and wounded, so that many more patients attended, some as out-patients.

Twenty-five cases have been recorded at this hospital, eight in officers and seventeen in other ranks. One patient only was a naval officer, and it is uncertain whether he was typical. The others were collected from all branches of the Army, and included one South African negro driver. All Army cases commenced in the Western Desert or Libya, with three exceptions, i.e., one started in Syria, one in the canal area, and one officer stated that he had habitually suffered in the same way on manoeuvres in India before the war, though the condition had been unrecognised and diagnosed as astigmatism.

A description of only twenty-five cases probably leads to underestimation of the prevalence of the disease. Captain Martin Williamson, M.C., R.A.M.C., stated in a private communication

- Received for publication, Novamber 22, 1943. 
that there was an epidemic of eye complaints which reached serious proportions in the first half of July and during which as much as 10 per cent. of men were suffering. Despite the fact that he had no facilities for examination, he gives such an accurate account of the symptoms and macroscopic signs that there is no doubt that the condition was widespread. He also noted that it occurred especially in observation post officers, drivers, and in patrols-all key men. Therefore the condition is of greater practical importance than this small series suggests.

Symptoms. - These are characteristic and constant. Most intelligent patients described an abrupt onset and could describe the symptoms accurately.

These are (1) smarting and itching of one or both eyes; (2) profuse lacrymation; (3) marked photophobia; and (4) definite disturbance of vision, less frequently described but noted among the more intelligent. This is either a uniocular diplopia, in which - a fainter image is seen either above or below an object, such as a distant aeroplane or tank, or else such dark objects have a tail-like prolongation like a comet, or they are vaguely blurred. Where both eyes notice the defect both are found to be affected. This symptom is very definite in some cases where previously eyesight has been excellent, and in several cases the patient has just called with a view to getting spectacles.

Sometimes the symptoms are intermittent, lasting acutely for about three days, clearing up almost completely and then reappearing again. An important point is that the onset, when noted accurately, has been during the day.

Many of the cases, especially ${ }^{-}$those commencing during the fighting around Tobruk in June, were associated with a very marked state of physical and nervous exhaustion.

Objective findings.-These again are very constant. Examination was hindered by lack of a corneal microscope, so that exact localisation of the affected layer of the cornea was uncertain, but from the appearance and translucency of the lesion, and the healing without scarring, it was deduced that only the superficial epithelial layers were affected. This observation has been confirmed by the use of a slit-lamp in the examination of a recent case.

In the active cases there was a marked reddening of the exposed areas of the scleral conjunctiva and a moderate ciliary flush and irritability of the eyes. Although an episcleral injection is a commonplace amongst the desert troops, in these cases it seemed more marked than usual, and it diminished greatly as the cornea healed under treatment.

The corneal changes were of three main types :-

(1) There was a bandlike roughening of the corneal epithelium across the area which would be exposed when the eyes are partially 
screwed up as is the case in a bright light. The band was greyish, but translucent and stained with fluorescein, revealing the loss of epithelium. The appearance was punctate. This was the commonest lesion found (Fig. 1).

(2) The lesion was sometimes limited to a few lines of greyish dots, mainly horizontal and in the exposed regions of the cornea. These dots also stained with fluorescein (Fig. 2).

(3) A single stippled line, actively staining, was also seen at times (Fig.3).

In two cases, where the history was very suggestive of this condition and the irregular astigmatism was noted, corneal lesions of the type of Fig. 4 were seen. There was no opacity in the central corneal area, but superficial stippling was seen at the limbal part of the " exposure area." As these cases responded to treatment just as the typical ones did, it is probably correct to regard them as a variety of the condition.

Commencement of healing ' was shown by the failure of fluorescein to stain the lesions. Some cases of the type of Fig. 1 which were treated throughout were seen to pass through stages corresponding to Figs. 2 and 3 in turn. It is uncertain, therefore, whether Figs. 2 and 3 represent later stages of the condition which starts as in Fig. 1, or whether the condition may commence with the smaller areas of corneal damage. Only examination of cases at the commencement of symptoms will elucidate this point.

On retinoscopy, patients having markedly defective vision were found to have a gross distortion of the retinal reflex. Sometimes the distortion could be seen to be directly due to the band-like lesions; in other cases, especially the Fig. 4 type, the cornea appeared to be clear, but the reflex was wildly irregular, or of "scissors" type. Not only was the marked reflex irregularity present, but it was impossible to correct the affected eyes with lenses subjectively.

The visual acuity improved pari passu with the healing. In the active state acuity was often reduced to $6 / 18$ or $6 / 12$. It returned to $6 / 6$ or $6 / 5$ unaided where this had been the previous visual standard, as the condition recovered.

The other parts of the eyes appeared unaffected, and no changes have been noted in lens or retina in any case.

Treatment.-Most cases, especially uniocular ones, have been treated with bandaging of the affected eye to give maximal rest to the cornea. Others where the condition was bilateral have probably done as well with the instillation of castor or cod-liver oil.

Full mydriasis relieves the discomfort rapidly and may hasten healing. Owing to service conditions it has only been possible to follow-up relatively few cases, but they have done uniformly 


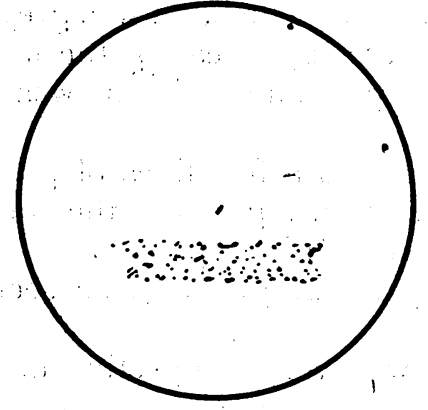

Fig. 1.

Band-like epithelial loss, staining with fluorescein, a common first stage.

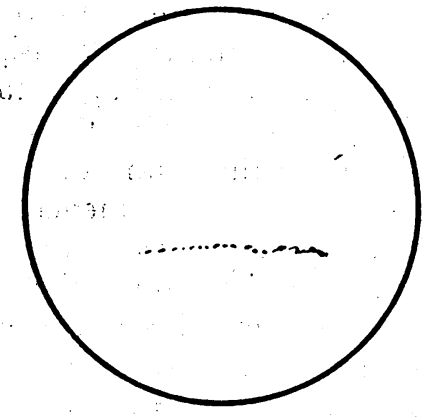

FIG. 3.

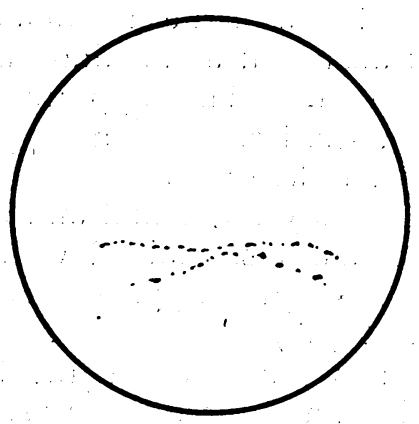

FIG. 2.

Linear punctate epithelial damage. Staining with fluorescein. Sometimes seen as a first stage and sometimes a healing stage

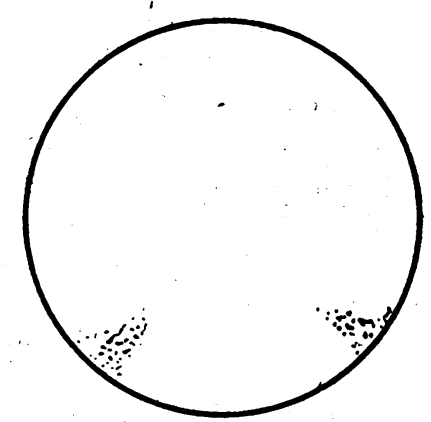

FIG. 4.

A single stippled line. Not always A less common corneal change. Not staining with fluorescein. Seen some- seen staining actively. times on first admission to hospital. Sometimes a healing stage.

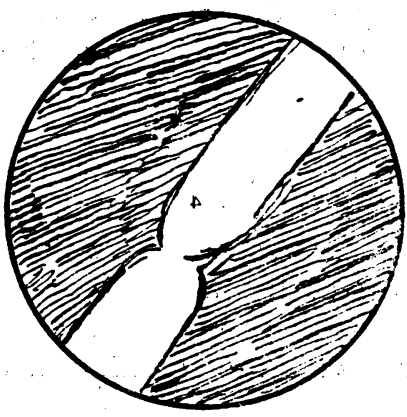

Fig. 5.

Distortion of the rotinal reflex as seen with Hamblin's streak retinoscope. Condition correspopding to types $1-3$. 


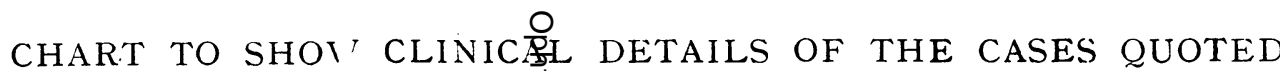

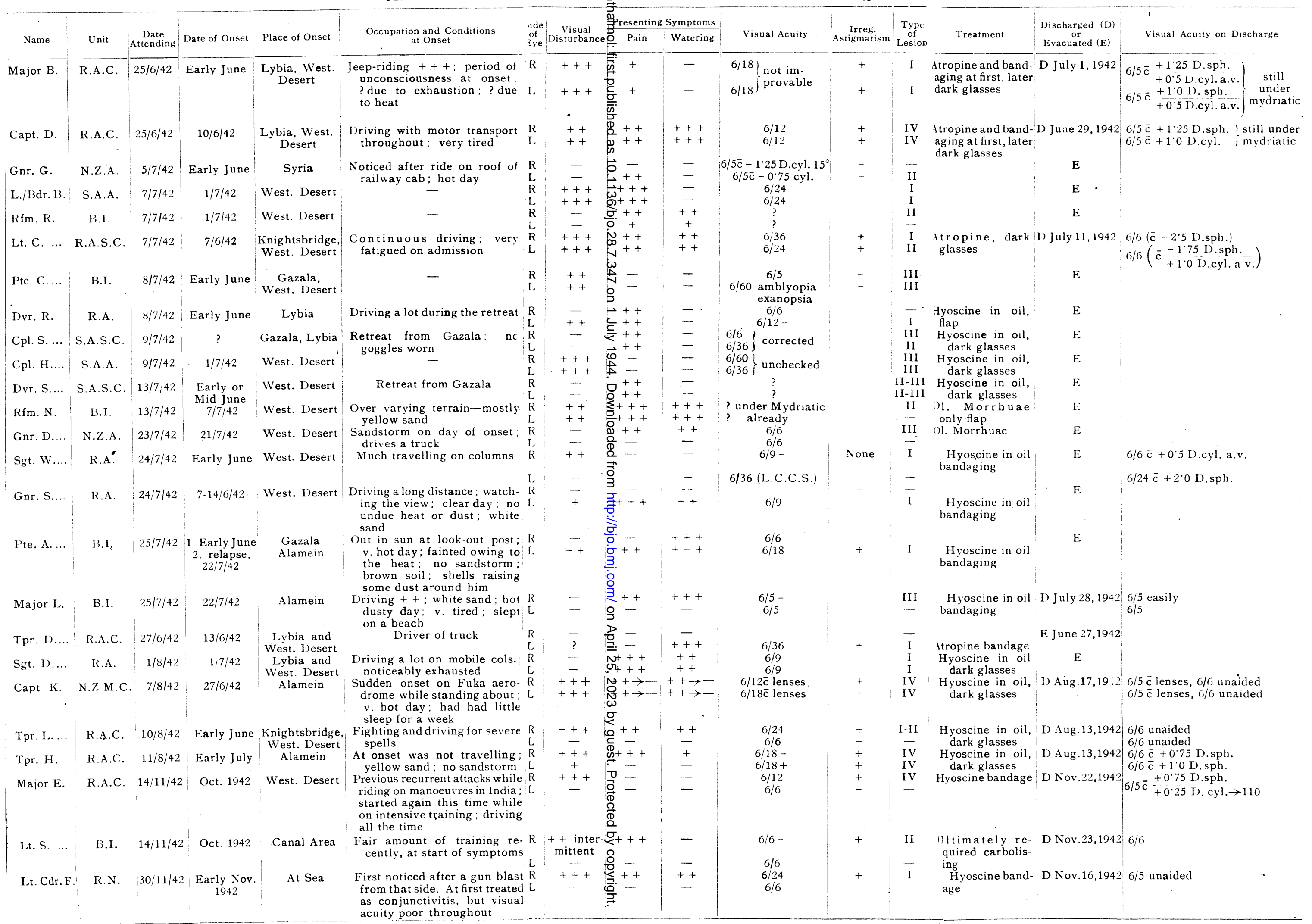


well. In all except one case the cornea has been healed and clear, and the visual acuity restored to normal in between five and seven days. The patients have been fit for duty, and those of whom news has been received have remained well and active since their return to the desert.

Discussion of Aetiology.-Three points strongly suggest that the lesion was caused by some physical factor :-

(1) The invariable localisation of the lesion to the small area of the cornea exposed by the eyelids when screwed up as protection against the bright sunlight of the desert.

(2) Its resemblance to the results of "flash" burns affecting the eyes and the vesiculation, caused by ultra-violet light emanations acting on the cornea, and to keratitis e lagophthalmo.

(3) The fact that the majority of the patients gave a history of driving in open vehicles just before or at the start of the symptoms.

The case which arose in Syria was in a soldier who travelled on a very long train journey on a hot day, sitting outside the railway carriage.

In another patient, an artilleryman, the condition started at the end of a drive from Mersa Matruh to Halfaya Pass, in which he had been admiring the scenery approaching the famous pass by looking over the top of the driver's cab.

A minority of the patients did not give a history of driving, but had been in a very exposed position in the desert and had noticed the heat unduly.

In one case, the naval officer, the onset of his visual disturbance and inflammation one month previously, which was diagnosed as conjunctivitis at first, was just after receiving the blast of a gun on the same side as the affected eye. He was found to have the corneal change of Fig. 1 type and irregular astigmatism of a typical case, and therefore he is included in the list, although he was not exposed to the desert conditions.

Discussions of the carse of the lesions.-The literature available shows that a similar condition has been described in cases of snow-blindness. Würdemann ("Injuries of the Eyes:") mentions the presence of a corneal erosion in this condition. G. L. Struder (Ophthal. Rec., November, 1906) recorded six cases of snow-blindness occurring during a blizzard in North America, where a corneal erosion could only be found by staining with fluorescein. He mentions that Graddy, of Nashville, Tenn., had previously described a similar condition due to exposure on snow fields. These observers ascribed the condition to ultra-violet emanations reflected from the snow.

In this connexion, a very good description of the condition has been given by Halphen (and Sedan), who noted a practically 
horizontal opacity " keratite en bandelettes" and stainable by fluorescein. The opacity remained for ten days. They say " one might call the superficial alterations. 'steamy cornea'-buée epitheliale."

Duke-Elder, in describing photophthalmia ("Rec. Adv. in Ophth."), writes that it may be caused by sunlight when reinforced by surfaces such as sea, desert, or snowfield.

In the investigation of photophthalmia produced experimentally the first experiments were made by Widmark (1889-1903), using an arc-light, which produced a reaction in the epithelial cells and to a lesser degree in the substantia propria of the cornea. With heavy doses lenticular opacities were produced. BirchHirschfeld,Kertel, Breose, Ognell, and Strebel found only corneal but no lenticular changes. Verhoeff and Bell, Hess and DukeElder have, however, produced lenticular changes.

In view of the changes found in experimental and clinical photophthalmia it has been assumed that desert and snow-blindness are due to the action of ultra-violet light reflected into the eyes.

In the literature at my disposal no description has been found of any similar condition occurring in a desert, and no mention has been made of the effect of hot air or wind playing on the exposed surface of the eye.

Possible Causative Factors.-The following may be considered :

(1) Damage by light radiations of short wave-length, i.e., photophthalmia.

(2) Damage of the epithelium by driven sand, either in a sandstorm or raised by vehicles driving in front or by shelling.

(3) The action of irritant gases.

(4) Drying of the exposed area of the cornea by intensely hot air or wind.

(5) Undue exposure of the affected area of the cornea by fatigue, which reduces the frequency of the blinking reflex of the lids. This would predispose to factor 4 .

Vitamin deficiency may be ruled out, as these patients have. all been in good bodily condition and showing no sign of other defects, apart from physical exhaustion in several cases.

(1) Photophthalmia.-Three points are strongly against this being the cause. First, the outstanding feature of clinical photophthalmia is the well-marked latent period between exposure and development of symptoms, corrobørated by Duke-Elder's experimental irradiation of the corneae of rabbits. In quite a large proportion of the cases under review, and especially noticeable in those men who gave an intelligent history, the onset of the symptoms was during the period of exposure.

Secondly, since the soil of different parts of the desert varies 
considerably in colour, one would have expected the whiter sands of the El Alamein area to reflect more ultra-violet light than the browner soil of the Tobruk area. Actually more cases occurred around Tobruk than in any other single area.

Thirdly, no cases have been noted in the Mediterranean Fleet, where the conditions of service undoubtedly expose the eyes of the sailors to a great deal of radiation.

It is improbable, therefore, that this condition is a type of photophthalmia.

(2) Damage by sand.-Although dust was blamed by many victims, in at least three cases it could not have been a factor. These were the men who developed it on the train in Syria, the gunner who was admiring the view of Halfaya, and the naval patient who received the blast of the gun. Other cases have also stated that they did not consider it unduly dusty when the condition started. Where patients have ascribed the onset to a sandstorm they have also admitted that it was a day of particular heat (a typical weather combination in Egypt). Indeed, one patient did have treatment for heat-stroke on the day that the symptoms started, and another also stated that he had " passed out " for some hours from an unknown cause, either the heat or exhaustion, on the date of the onset.

It is impossible to find enough cases to suggest that sand is not an invariable factor.

It is noteworthy that in no case was any sand particle found embedded in the cornea, nor were any ulcers seen suggestive of this having occurred, and, again, foreign particles in the conjunctival sac do sometimes produce tiny superficial abrasions where the lids rub them against the corneal epithelium. In these cases the line of each individual scratch appears to be vertical, whereas in the keratitis described the spacing of individual grey dots in the band-like area affected was quite regular and not arranged in vertical linear bars,

The general appearance of the Iesion was so similar to those resulting from cocaine, exposure to air and flame, that one could not help feeling that sand was an unlikely cause by comparison.

(3) Irritant Gases. - In no case could a history of exposure to fumes of any sort be elicited, apart from the smoke of shells in a few cases.

(4) Drying of the cornea.-The appearance of the lesion being that of one with a physical cause leads to the conclusion that drying of the cornea by the hot, dry air of the desert is the probable cause. Three points strongly favour this hypothesis:-

(1) The majority of the patients have been riding open vehicles at the start of the symptoms.

(2) In no case in the records have glasses or goggles been worn at the time of onset. 
(3) This is the first occasion on which there has been prolonged and hard fighting in the hottest part of the year, with short nights, permitting little sleep.

(5). Undue exposure of the cornea from fatigue. - It was most noticeable that these patients seen in the early stages were extremely fatigued, and looked mere shadows of the men they became after three days in hospital. The officers and N.C.O.s, who were of an energetic and keen type, admitted that before the start of the symptoms " things had been pretty hectic," to use a typical understatement. 'It is also noteworthy that, with one or two exceptions, all the men had come from forward areas, the majority being from fighting units who had been in the thick of the fray.

It is possible that in cases of great fatigue blinking may be reduced in frequency. This reflex is a most important factor. in moistening the cornea, and if it were reduced would pre-dispose to drying.

\section{Discussion}

From a consideration of the above points it is probable that the physical cause of the lesion is drying of the corneal epithelium. The weather conditions in North Africa are such that the average temperature rises as the summer progresses, but in the early part of the summer spells of southerly winds with high temperature and low humidity are frequent. During these spells the wind has a great drying effect on the skin and feels noticeably hot as it blows on the eyeballs.

It is possible that similar lesions, ascribed to photophthalmia when occurring on snowfields, are again at least in part due to desiccation and perhaps to freezing. as well. One or two men who claimed to have suffered from "snow-blindness" in the Archangel expedition of 1919 have been interrogated, and stated that the onset was during long drives and that there was no latent period between exposure and the onset of symptoms. This suggests that "snow-blindness" may not, in some cases at least, behave like a true photophthalmia, and that it may have been wrong to ascribe the band-like corneal opacity to the effect of ultraviolet light radiations.

(1) Temporary damage has been caused to the eyes of soldiers serving in the North African desert. The site is the corneal epithelium and the condition is transient.

(2) Discomfort, photophobia and impairment of vision are the outstanding symptoms.

(3) It is probable that the cause is drying of the corneal epithelium by a hot, dry wind, which causes excessive evaporation of the lacrymal secretion over the exposed area of the cornea.

(4) The suggestion is put forward that corneal damage ascribed 
to snow-blindness is really caused, certainly in part, by desiccation and perhaps also freezing of the corneal epithelium, and not by excessive ultra-violet light reflections.

(5) The condition could be prevented by the wearing of gogglés.

(6) The name desiccation keratitis is suggested for the condition.

I would like to express my gratitude to Brigadier C. W. Graham and Brigadier G. I. Scott and to Dr. Rowland Wilson, of the Giza Memorial Hospital, Cairo, for their great help and advice in the preparation of this paper, and to Colonel $\mathrm{J}$. Morrison, O.B.E., M.C., for permission to publish it.

\title{
THE SECRETION-DIFFUSION THEORY OF INTRA-OCULAR FLUID DYNAMICS*
}

BY

\author{
V. Everet T Kinsey and W. Morton Grant \\ HOWE LABORATORY OF OPHTHALMOLOGY, HARVARD UNIVERSITY \\ MEDICAL SCHOOL, BOSTON, MASSACHUSETTS
}

Recentiy Duke-Elder and .Davson ${ }^{1}$ discussed certain phases of our paper entitled " The mechanism of aqueous humour formation inferred from chemical studies on blood-aqueous humour dynamics" 2. From their comments it is apparent that these authors obtained the wrong impression concerning our viewpoint, Perhaps as a consequence they incorrectly modified certain equations which we had set up to represent the ultra-filtration mechanism of aqueous humour formation. We feel therefore that some recapitulation of our theory of intra-ocular fluid dynamics is in order. At the same time we wish to discuss the criticisms which DukeElder and Davson have made concerning the mathematical concepts we have used in developing our theory.

Through the use of heavy water $\left(\mathrm{D}_{2} \mathrm{O}\right)$ as a tracer substance for ordinary water it was shown that one half of the water in the anterior chamber of the rabbit was replaced in slightly less than three minutes ${ }^{3}$. This is equal to a total water movement into and out of the anterior chamber of approximately fifty cubic millimetres per minute, a rate, it will be observed, far in excess of that which was thought to correspond to the rate of formation of. aqueous humour as a whole. This finding suggested that the various constituents of the aqueous humour may enter the anterior chamber at different rates, and that it was unrealistic therefore, to speak of a rate of formation of aqueous humour as a whole. To

* Received for publication, February 4, 1944. 